Pacific Journal of Mathematics

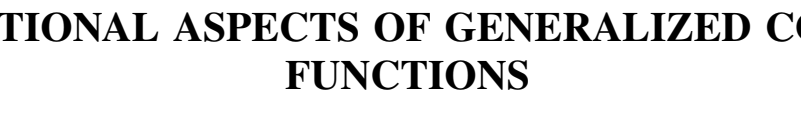




\title{
VARIATIONAL ASPECTS OF GENERALIZED \\ CONVEX FUNCTIONS
}

\author{
William T. REID
}

1. Introduction. For a second order linear homogeneous differential equation

$$
L(y) \equiv y^{\prime \prime}+p_{1}(x) y^{\prime}+p_{2}(x) y=0,
$$

with $p_{1}(x), p_{2}(x)$ continuous real-valued functions on an open interval $(a, b)$ of the real line, and such that for arbitrary $x_{1}, y_{1}, x_{2}, y_{2}$ with $a<x_{1}<x_{2}<b$ there is a unique solution $y(x)=y\left(x ; x_{1}, y_{1} ; x_{2}, y_{2}\right)$ of (1.1) satisfying $y\left(x_{\alpha}\right)=y_{\alpha},(\alpha=1,2)$, a real-valued function $u(x)$ has been termed "sub- $(L)$ on $(a, b)$ " if for arbitrary $c$, $d$ on $a<c<d<b$ we have

$$
u(x) \leqq y(x ; c, u(c) ; d, u(d)) \text { on } c \leqq x \leqq d .
$$

The class of such sub- $(L)$ functions is a special instance of sub- $F$ functions as introduced by Beckenbach [1], who established for general sub- $F$ functions various properties analogous to those of convex functions.

In particular, for sub- $(L)$ functions it has been established by Peixoto [8] and Bonsall [3] that a real-valued function $u(x)$ of class $C^{\prime \prime}$ on $(a, b)$ is $\operatorname{sub}-(L)$ on this interval if and only if $L(u) \geqq 0$ on $(a, b)$; indeed, Peixoto has shown that for certain types of non-linear second order differential equations the corresponding sub-functions of class $C^{\prime \prime}$ are characterized by a similar differential inequality. Now if $a<x_{0}<b$ and

$$
r_{0}(x)=\exp \left[\int_{x_{0}}^{x} p_{1}(t) d t\right], p_{0}(x)=-p_{2}(x) r_{0}(x),
$$

then for a function $u(x)$ of class $C^{\prime \prime}$ the condition $L(u) \geqq 0$ on $(a, b)$ is equivalent to the condition that on each compact subinterval $[c, d]$ of $(a, b)$ the function $u(x)$ affords a minimum to the integral

$$
\int_{c}^{a}\left[r_{0}(x) y^{\prime 2}+p_{0}(x) y^{2}\right] d x
$$

in the class of $y(x)$ that are absolutely continuous with $y^{\prime}(x)$ of integrable square on $[c, d]$, and

$$
y(c)=u(c), y(d)=u(d), y(x) \leqq u(x) \text { on }[c, d] .
$$

Received August 20, 1958. These results were obtained while the author was visiting professor at the University of California, Los Angeles. This paper was prepared in part under the sponsorship of the Office of Naval Research. Reproduction in whole or in part is permitted for any purpose of the United States Government. 
It is the purpose of the present paper to show that sub- $(L)$ functions in general are characterized by the property of affording a minimum for an associated unilateral variation problem. For such a study it is more appropriate to consider the differential equation in self-adjoint form, and such is done throughout the paper.

Certain preliminary results on sub- $(L)$ functions are presented in $\S 2$; $\S 3$ is devoted to variational criteria of the type mentioned above, and related results. Finally, in $\S 4$ it is shown that sub- $(L)$ functions are characterized by a property that is a direct generalization of the wellknown fact that a real-valued function is convex on $(a, b)$ if and only if $u(x)$ is continuous and

$$
u(x) \leqq(2 h)^{-1} \int_{x-h}^{x+h} u(t) d t
$$

for all $x \in(a, b)$ and $h>0$ such that $[x-h, x+h]$ is a subinterval of $(a, b)$.

In regard to similar problem involving partial differential equations in two independent variables, it is to be commented that Levin [6] has considered the minima of double integrals with respect to unilateral variations, with special attention to subharmonic functions; for more general multiple integrals necessary conditions for a unilateral variation problem are given by Carson [4; Sections 8, 10].

2. Prefatory results. Suppose that $r(x), p(x)$ and $q(x)$ are real-valued continuous functions of the real variable $x$ on the open interval $(a, b)$ with $r(x)>0$ on this interval, and consider the self-adjoint differential equation

$$
L(y) \equiv\left(r(x) y^{\prime}+q(x) y\right)^{\prime}-\left(q(x) y^{\prime}+p(x) y\right)=0 .
$$

By a solution of $(2.1)$ is meant a $y(x)$ such that on $(a, b)$ the functions $y(x)$ and $r(x) y^{\prime}(x)+q(x) y$ are continuously differentiable and $L(y) \equiv 0$. We shall be concerned with equations (2.2) which possess the following property:

(I). If $a<x_{1}<x_{2}<b$ and $y_{1}, y_{2}$ are arbitrary real numbers, then there exists a unique solution $y(x)=y\left(x ; x_{1}, y_{1} ; x_{2}, y_{2}\right)$ of $(2.1)$ such that $y\left(x_{\alpha}\right)=y_{\alpha},(\alpha=1,2)$.

Corresponding to the terminology of Bonsall [3] and Peixoto [8], a function $u(x)$ is termed "sub- $(L)$ on $(a, b)$ " if $u(x)$ is real-valued, and for arbitrary $x_{1}, x_{2}$ satisfying $a<x_{1}<x_{2}<b$ we have

$$
u(x) \leqq y\left(x ; x_{1}, u\left(x_{1}\right) ; x_{2}, u\left(x_{2}\right)\right) \text { on } x_{1} \leqq x \leqq x_{2} .
$$

A function $u(x)$ is said to be "strictly sub- $(L)$ on $(a, b)$ " if for arbitrary $x_{1}, x_{2}$ satisfying $a<x_{1}<x_{2}<b$ the strict inequality holds in (2.2) for $x_{1}<x<x_{2}$. 
LEMMA 2.1. Condition (l) implies that there exists a solution $y_{0}(x)$ of (2.1) such that $y_{0}(x)>0$ on $(a, b)$; moreover, if $a<x_{0}<b$ and $x=x[t]$ is defined by

$$
t=\int_{x_{0}}^{x} \frac{d z}{y_{0}^{2}(z) r(z)}, \quad \alpha<t<\beta,
$$

where

$$
\alpha=\lim _{x \rightarrow a} \int_{x_{0}}^{x} d z /\left[y_{0}^{2}(z) r(z)\right], \beta=\lim _{x \rightarrow b} \int_{x_{0}}^{x} d z /\left[y_{0}^{2}(z) r(z)\right],
$$

then $u(x)$ is sub- $(L)$ on $(a, b)$ if and only if $\tilde{u}(t)=u(x[t]) / y_{0}(x[t])$ is convex on $(\alpha, \beta)$.

If $a<x_{0}<b$, and $a<s<b, s \neq x_{0}$, then condition (I) implies the existence of a unique solution $y(x ; s)$ of $(2.1)$ satisfying $y\left(x_{0} ; s\right)=1$, $y(s ; s)=0$. In view of condition (I) it follows readily that

$$
\begin{gathered}
y^{\prime}\left(x_{0} ; s_{1}\right)<y^{\prime}\left(x_{0} ; s_{2}\right), y\left(x, s_{1}\right)<y\left(x, s_{2}\right) \text { on } x>x_{0}, \text { if } x_{0}<s_{1}<s_{2} ; \\
y^{\prime}\left(x_{0} ; s_{1}\right)<y^{\prime}\left(x_{0} ; s_{2}\right), y\left(x, s_{1}\right)>y\left(x, s_{2}\right) \text { on } x<x_{0}, \text { if } s_{1}<s_{2}<x_{0} ; \\
y^{\prime}\left(x_{0} ; s_{2}\right)<y^{\prime}\left(x_{0} ; s_{1}\right), \text { if } s_{1}<x_{0}<s_{2} .
\end{gathered}
$$

Consequently, $\quad k_{b}=\lim _{s \rightarrow b} y^{\prime}\left(x_{0} ; s\right)$ and $k_{a}=\lim _{s \rightarrow a} y^{\prime}\left(x_{0} ; s\right)$ are finite, $k_{b} \leqq k_{a}$, and if $y_{0}(x)$ is a solution of (2.1) satisfying the initial conditions $y_{0}\left(x_{0}\right)=1, k_{b} \leqq y_{0}^{\prime}\left(x_{0}\right) \leqq k_{a}$, then $y_{0}(x)>0$ on $(a, b)$.

In view of the identity

$$
y_{0}(x) L\left(y_{0} w\right) \equiv\left(y_{0}^{2}(x) r(x) w^{\prime}\right)^{\prime}
$$

it follows that if $x[t]$ is defined by (2.3) then $y(x)$ is a solution of (2.1) if and only if $\tilde{y}(t)=y(x[t]) / y_{0}(x[t])$ is a linear function of $t$ on $(\alpha, \beta)$. Moreover, it is clear that a function $u(x)$ is sub- $(L)$ on $(a, b)$ if and only if $\tilde{u}(t)=u(x[t]) / y_{0}(x[t])$ is a convex function on $(\alpha, \beta)$, that is, $\tilde{u}(t)$ is $\operatorname{sub}-\left(L_{0}\right)$ on $(\alpha, \beta)$ with $L_{0}(w) \equiv w^{\prime \prime}$.

LEMMA 2.2. If $u(x)$ is sub-(L) on $(a, b)$, then for an arbitrary compact sub-interval $[c, d]$ of $(a, b)$ :

(i) $u(x)$ is Lipschitzian on $[c, d]$;

(ii) if $\Pi$ is a partition $c=x_{0}<x_{1}<\cdots<x_{n}=d$, ( $\left.n \geqq 2\right)$, of $[c, d]$, and $y_{\Pi 1}(x)$ is the continuous function on $[c, d]$ defined by

$$
y_{\Pi}(x)=y\left(x ; x_{j-1}, u\left(x_{j-1}\right) ; x_{j}, u\left(x_{j}\right)\right), x_{j-1} \leqq x \leqq x_{j}, \quad(j=1, \cdots, n),
$$

then

(a) at $x_{k},(k=1, \cdots, n-1)$, the right-and left-hand derivatives $y_{\Pi}^{\prime}\left(x_{k}^{+}\right)$and $y_{\Pi}^{\prime}\left(x_{k}^{-}\right)$satisfy $y_{\Pi}^{\prime}\left(x_{k}^{+}\right) \geqq y_{\Pi}^{\prime}\left(x_{k}^{-}\right)$;

(b) there exists a constant $M$ independent of II such that $\left|y_{11}^{\prime}(x)\right| \leqq M$ on $[c, d]$, and if $\|I\|$ denotes the maximum of $x_{j}-x_{j-1},(j=1, \cdots, n)$, 
then as $\|I\| \rightarrow 0$ we have $y_{\Pi}(x) \rightarrow u(x)$ uniformly on $[c, d]$ and $y_{\mathrm{II}}^{\prime}(x) \rightarrow$ $u^{\prime}(x)$ almost everywhere on this interval.

For the case $L(y) \equiv y^{\prime \prime}$ the results of this lemma are classical results on convex functions; for the general equation (2.1) they are corollaries of the corresponding results for convex functions, in view of Lemma 2.1 .

It is to be commented that the arguments used by Bonsall [3] to establish his Lemma 1, Theorems 1 and 2 may be employed to prove the same results for functions that are sub- $(L)$ with $L(y)$ of the form (2.1), and these results imply conclusions (i) and (iia) of the above lemma; moreover, it is not difficult to give a direct proof of conclusion (iib) that does not employ the auxiliary transformation of Lemma 2.1. It is to be remarked also that conclusion (i) of the lemma may be derived as a consequence of Theorem 3 of Green [5].

3. Variational criteria. If $a<c<d<b$, for brevity we shall denote by $\Gamma(c, d)$ the class of real-valued functions $\eta(x)$ that are absolutely continuous on the compact interval $[c, d]$, and $\eta^{\prime}(x)$ belongs to the class $2^{2}(c, d)$ of functions of Lebesgue integrable square on this interval. The symbol $\Gamma_{0}(c, d)$ will signify the class of functions $\eta(x) \in \Gamma(c, d)$ for which $\eta(c)=0=\eta(d)$, and $\Gamma_{0}^{-}(c, d)$ will denote the class of functions $\eta(x) \in \Gamma_{0}(c, d)$ satisfying $\eta(x) \leqq 0$ on $[c, d]$.

LemMA 3.1. Condition (I) holds if and only if for arbitrary $c$, d satisfying $a<c<d<b$ the quadratic functional

$$
I(\eta ; c, d) \equiv \int_{c}^{a}\left[r(x) \eta^{\prime 2}+2 q(x) \eta \eta^{\prime}+p(x) \eta^{2}\right] d x
$$

is positive definite on $\Gamma_{0}(c, d)$, that is, $I(\eta ; c, d) \geqq 0$ for $\eta \in \Gamma_{0}(c, d)$ and the equality sign holds only if $\eta(x) \equiv 0$ on $[c, d]$. Moreover, if condition $(I)$ holds then for $y(x)$ an arbitrary solution of (2.1) and $a<c<d<b$,

$$
I(\eta ; c, d) \geqq I(y ; c, d) \text { for } \eta-y \in \Gamma_{0}(c, d),
$$

and the equality sign in (3.2) holds only if $\eta(x) \equiv y(x)$ on $[c, d]$.

Condition (I) is clearly equivalent to the condition that (2.1) is nonoscillatory on $(a, b)$, that is, if $y(x)$ is a solution of (2.1) for which $y\left(x_{1}\right)=0=y\left(x_{2}\right)$, where $a<x_{1}<x_{2}<b$, then $y(x) \equiv 0$. In turn, the fact that non-oscillation of (2.1) on a subinterval $[c, d]$ is equivalent to the positive definiteness of $I(\eta ; c, d)$ on $\Gamma_{0}(c, d)$ is a well-known result of the calculus of variations, (see, for example, Bliss [2; Chapter IV], or Morse [7; Chapter I]).

Let $I\left(\eta_{1}, \eta_{2} ; c, d\right)$ denote the bilinear functional 


$$
I\left(\gamma_{1}, \quad \gamma_{2} ; c, d\right)=\int_{0}^{a}\left[\gamma \gamma_{1}^{\prime}\left(r \gamma_{j_{2}}^{\prime}+q \gamma_{2}\right)+\gamma_{1}\left(q \gamma_{/ 2}^{\prime}+p \gamma_{2}\right)\right] d x .
$$

If $L(y)=0$ and $\eta-y \in \Gamma_{0}(c, d)$ then

$$
I(\eta-y, y ; c, d)=\left[(\eta-y)\left(r y^{\prime}+q y\right)\right]_{c}^{a}=0,
$$

and the final statement of the theorem is an immediate consequence of the identity

$$
I(\eta ; c, d)=I(y ; c, d)+2 I(\eta-y, y ; c, d)+I(\eta-y ; c, d)
$$

and the previously established result that condition (I) implies $I(\eta-y ; c, d)>0$ unless $\eta(x) \equiv y(x)$ on $[c, d]$.

The central result of this paper is the following theorem.

THeORem 3.1. If (2.1) satisfies condition (I) then $u(x)$ is sub-(L) on $(a, b)$ if and only if for each compact subinterval $[c, d]$ of $(a, b)$ the function $u(x)$ belongs to $\Gamma(c, d)$, and

$$
I(\eta ; c, d) \geqq I(u ; c, d) \text { for } \eta-u \in \Gamma_{0}^{-}(c, d) .
$$

Before presenting a proof of this theorem, it is to be remarked that if condition $(I)$ holds and $u(x) \in \Gamma(c, d)$ then condition (3.3) is equivalent to

$$
I(\zeta, u ; c, \mathrm{~d}) \geqq 0 \text { for } \zeta \in \Gamma_{0}^{-}(c, d) .
$$

Indeed, by Lemma 3.1 condition $(I)$ implies $I(\zeta ; c, d) \geqq 0$ for $a<c<d<b$ and $\zeta(x) \in \Gamma_{0}(c, d)$, while for $\theta>0$ the function $\theta \zeta(x) \in \Gamma_{0}^{-}(c, d)$ whenever $\zeta(x) \in \Gamma_{0}^{-}(c, d)$, so that if $\zeta(x) \in \Gamma_{0}^{-}(c, d)$ the equivalence of (3.3) and $\left(3.3^{\prime}\right)$ is an immediate consequence of the identity

$$
I(u+\theta \zeta ; c, d)=I(u ; c, d)+2 \theta I(\zeta, u ; c, d)+\theta^{2} I(\zeta ; c, d) .
$$

Now suppose that $u(x)$ is such that for each compact subinterval $[c, d]$ of $(a, b)$ we have $u(x) \in \Gamma(c, d)$ and (3.3) holds. If $a<a_{1}<b_{1}<b$ and $y(x)=y\left(x ; a_{1}, u\left(a_{1}\right) ; b_{1} u\left(b_{1}\right)\right)$, then in case $u(x) \leqq y(x)$ does not hold throughout $\left[a_{1}, b_{1}\right]$ there exist values $c, d$ such that $a_{1} \leqq c<d \leqq b_{1}$ and $y(c)=u(c), y(d)=u(d), u(x)>y(x)$ on $(c, d)$. Then $y-u \in \Gamma_{0}^{-}(c, d)$ and (3.3) implies that $I(y ; c, d) \geqq I(u ; c, d)$, whereas Lemma 3.1 provides the contradictory result $I(u ; c, d)>I(y ; c, d)$. Hence $u(x) \leqq y(x)$ on $\left[a_{1}, b_{1}\right]$, and in view of the arbitrariness of $a_{1}, b_{1}$ we have that $u(x)$ is sub- $(L)$ on $(a, b)$.

On the other hand, if $u(x)$ is sub- $(L)$ on $(a, b)$ and $[c, d]$ is a subinterval of $(a, b)$, then by conclusion (i) of Lemma 2.1 the function $u(x)$ is Lipschitzian on $[c, d]$, and hence $u(x) \in \Gamma(c, d)$. If $\Pi: c=x_{0}<x_{1}<$ $\cdots<x_{n}=d$ is a partition of this subinterval, and $y_{\text {ПІ }}(x)$ is the function 
defined by (2.4), then $y_{11}^{\prime}\left(x_{k}^{+}\right)-y_{11}^{\prime}\left(x_{k}^{-}\right) \geqq 0, \quad(k=1, \cdots, n-1)$. Since $L\left(y_{\mathrm{n}}\right)=0$ on each subinterval $\left(x_{j-1}, x_{j}\right),(j=1, \cdots, n)$, if $\zeta(x) \in I_{0}^{-}(c, d)$ then $\zeta\left(x_{k}\right) \leqq 0,(k=1, \cdots, n-1)$, and an integration by parts yields

$$
I\left(\zeta, y_{\Pi} ; c, d\right)=\sum_{k=1}^{n-1} \zeta\left[r y_{\text {I }}^{\prime}+q y_{\mathrm{II}}\right]_{x=x_{k}^{+}}^{x=x_{k}^{-}} \geqq 0 \text { for } \zeta \in \Gamma_{0}^{-}(c, d) \text {. }
$$

If $\left\{\Pi_{m}\right\},(m=1,2, \cdots)$, is a sequence of partitions of $[c, d]$ such that $\left\|\Pi_{m}\right\| \rightarrow 0$, then from conclusion (iib) of Lemma 2.1 and the Lebesgue bounded convergence theorem it follows that (3.3') holds, and hence (3.3) is satisfied by this function $u(x)$.

It is to remarked that an alternate method of proof for the above theorem is to establish this result for the special case of convex functions, that is, for the special differential equation $L(y) \equiv y^{\prime \prime}=0$, and to reduce the general case to this special case by means of the transformation of Lemma 2.1.

In view of the linearity of $I(\zeta, u ; c, d)$ as a functional of $\zeta$, if for given $c, d$ we have $I(\zeta, u ; c, d)=0$ for all $\zeta \in \Gamma_{0}^{-}(c, d)$ then $I(\zeta, u ; c, d)=0$ for all $\zeta \in \Gamma_{0}(c, d)$, and from the fundamental lemma of the calculus of variations it follows that $u(x)$ is a solution of (2.1) on $(c, d)$. Since a sub- $(L)$ function $u(x)$ can fail to be strictly sub- $(L)$ only if there is a subinterval on which $u(x)$ is a solution of (2.1), we have the following result.

COROLLARY 1. If (2.1) satisfies condition (I) then $u(x)$ is strictly sub$L$ on $(a, b)$ if and only if for each subinterval $[c, d]$ of $(a, b)$ the function $u(x) \in \Gamma(c, d)$, inequality $\left(3.3^{\prime}\right)$ holds, and there is a function $\zeta \in \Gamma_{0}^{-}(c, d)$ such that the strict inequality in (3.3) holds.

Now for $u(x) \in \Gamma(c, d)$ an integration by parts yields

$I(\zeta, u ; c, d)$

$=\int_{a}^{b} \zeta^{\prime}(x)\left[r(x) u^{\prime}(x)+q(x) u(x)-\int_{x_{0}}^{x}\left(q(s) u^{\prime}(s)+p(s) u(s)\right) d s\right] d x$ for $\zeta \in \Gamma_{0}(c, d)$,

where $x_{0}$ is an arbitrary point on $(a, b)$. Consequently, if $u(x) \in \Gamma(c, d)$ for arbitrary subintervals [c, d] of $(a, b)$, it follows from Theorem 3.1 for the differential equation $L_{0}(y) \equiv y^{\prime \prime}=0$ that $u(x)$ satisfies $\left(3.3^{\prime}\right)$ for arbitrary subintervals $[c, d]$ if and only if the function

$$
\int_{x_{0}}^{x}\left[r(t) u^{\prime}(t)+q(t) u(t)-\int_{x_{0}}^{t}\left(q(s) u^{\prime}(s)+p(s) u(s)\right) d s\right] d t
$$

is convex on $(a, b)$, and we have the following result.

CoRollary 2. If (2.1) satisfies condition $(I)$ then $u(x)$ is sub- $(L)$ on $(a, b)$ if and only if $u(x) \in \Gamma(c, d)$ for arbitary compact subintervals $[c, d]$ of $(a, b)$, and (3.4) is a convex function on $(a, b)$; in particular, 
if $u(x)$ and $r(x) u^{\prime}(x)+q(x) u(x)$ are continuously differentiable on $(a, b)$ then $u(x)$ is sub- $(L)$ if and only if $L(u) \geqq 0$ on this interval.

In view of the linearity of $I(\zeta, u ; c, d)$ as a functional of the coefficients of $L(y)$, the results of the following corollaries are immediate consequences of the criteria of Lemma 3.1 and Theorem 3.1.

Corollary 3. If $L(y)$ satisfies condition $(I)$ on $(a, b)$, and $k(x)$ is a non-negative continuous function on this interval, then $L_{1}(y)=L(y)-$ $k(x) y$ satisfies $(I)$ on $(a, b)$, and if $u(x)$ is sub- $(L)$ on $(a, b)$ with $u(x) \leqq 0$ on a subinterval $\left(a_{1}, b_{1}\right)$, then $u(x)$ is sub- $\left(L_{1}\right)$ on $\left(a_{1}, b_{1}\right)$.

Corollary 4. If $r_{\alpha}(x), p_{\alpha}(x), q_{\alpha}(x),(\alpha=1,2)$, are real-valued continuous functions with $r_{a}(x)>0$ on $(a, b)$, and

$$
L_{\alpha}(y) \equiv\left(r_{\alpha}(x) y^{\prime}+q_{\alpha}(x) y\right)^{\prime}-\left(q_{\alpha}(x) y^{\prime}+p_{\alpha}(x) y\right)=0, \quad(\alpha=1,2),
$$

satisfy condition $(I)$ on $(a, b)$, then $L_{3}(y) \equiv L_{1}(y)+L_{2}(y)=0$ satisfies condition $(I)$ on this interval; moreover, if $u(x)$ is sub- $\left(L_{\alpha}\right),(\alpha=1,2)$, on $(a, b)$ then $u(x)$ is sub- $\left(L_{3}\right)$ on this interval.

4. Another characterization of sub- $(L)$ functions. It will be established now that sub- $(L)$ functions are characterized by a property that is a direct generalization of the well-known fact that a function $u(x)$ is convex on $(a, b)$ if and only if $u(x)$ is continuous and

$$
u(x) \leqq(2 h)^{-1} \int_{x-h}^{x+h} u(t) d t
$$

for all $x \in(a, b)$ and $h>0$ such that $[x-h, x+h]$ is a subinterval of $(a, b)$.

Suppose that (2.1) satisfies condition (I) on $(a, b)$, and for a given $s$ of this interval let $z=z(x ; s)$ be the solution of the system

$$
L(z)=-1, z(s)=0=z^{\prime}(s) .
$$

Since $r(x)>0$, it follows readily from $(4.2)$ that $z(x ; s)<0$ for $x$ in a sufficiently small deleted neighborhood of the point $s$. Moreover, by Corollary 2 to Theorem 3.1 the function $u(x)=-z(x ; s)$ is sub- $(L)$ on $(a, b)$; consequently, there is no subinterval $[c, d]$ such that $z(c ; s)=$ $0=z(d ; s), z(x ; s)<0$ on $(c, d)$, and hence $z(x ; s)<0$ for $x \neq s$. As the difference of two solutions of $L(z)=-1$ is a solution of (2.1), in view of condition (I) it follows that if $a<s_{1}<s_{2}<b$ then there is a unique value $\xi=\xi\left(s_{1}, s_{2}\right)$ such that $z\left(\xi ; s_{1}\right)=z\left(\xi ; s_{2}\right)$, and

$$
s_{1}<\xi\left(s_{1}, s_{2}\right)<s_{2}, z^{\prime}\left(\xi\left(s_{1}, s_{2}\right) ; s_{2}-z^{\prime}\left(\xi\left(s_{1}, s_{1}\right) ; s_{1}\right)>0 .\right.
$$

Consequently, if for $a<s_{1}<s_{2}<b$ we define $w_{s_{1} s_{2}}(x)$ as 


$$
\begin{aligned}
w_{s_{1} s_{2}}(x) & =z\left(x, s_{1}\right), s_{1} \leqq x \leqq \xi\left(s_{1}, s_{2}\right), \\
& =z\left(x, s_{2}\right), \quad \xi\left(s_{1}, s_{2}\right) \leqq x \leqq s_{2},
\end{aligned}
$$

we have that $w_{s_{1} s_{2}}(x) \in \Gamma_{0}^{-}\left(s_{1}, s_{2}\right)$ and

$$
k\left(s_{1}, s_{2}\right) \equiv r\left(\xi\left(s_{1}, s_{2}\right)\right)\left[w_{s_{1} s_{2}}^{\prime}\left(\xi\left(s_{1}, s_{2}\right)^{+}\right)-w_{s_{1} s_{2}}^{\prime}\left(\xi\left(s_{1}, s_{2}\right)^{-}\right)\right]>0 .
$$

Now if $y(x)$ is a solution of (2.1) then $I\left(w_{s_{1} s_{2}}, y ; s_{1}, s_{2}\right)=0$, and as $w_{s_{1} s_{2}}(x)=w_{s_{1} s_{2}}^{\prime}(x)=0$ at $x=s_{\alpha},(\alpha=1,2)$, an integration by parts yields the relation

$$
0=y(x)\left[r(x) w_{s_{1} s_{2}}^{\prime}(x)+q(x) w_{s_{1} s_{2}}(x)\right]_{x=\xi^{+}}^{x=\xi^{-}}-\int_{s_{1}}^{s_{2}} y L\left(w_{s_{1} s_{2}}\right) d x
$$

which may be written as

$$
y\left(\xi\left(s_{1}, s_{2}\right)\right)=\left[k\left(s_{1}, s_{2}\right)\right]^{-1} \int_{s_{1}}^{s_{2}} y(t) d t, \quad a<s_{1}<s_{2}<b .
$$

If $y_{0}(x)$ is any solution of (2.1) that is different from zero on $\left(s_{1}, s_{2}\right)$, then

$$
k\left(s_{1}, s_{2}\right)=\left[y_{0}\left(\xi\left(s_{1}, s_{2}\right)\right)\right]^{-1} \int_{s_{1}}^{s_{2}} y_{0}(t) d t
$$

in particular, uniformly for subintervals $\left[s_{1}, s_{2}\right]$ one may choose $y_{0}(x)$ as the function appearing in Lemma 2.1.

Now if $u(x)$ is $\operatorname{sub}-(L)$ on $(a, b)$, and $a<\xi<b$, there is a solution $y(x)$ of $(2.1)$ such that $y(\xi)=u(\xi), y(x) \leqq u(x)$ on $(a, b)$; moreover, if $u(x)$ is strictly sub- $(L)$ on $(a, b)$ then $y(x)<u(x)$ for $x \neq \xi$. This fact is a ready consequence of the corresponding result for convex functions and Lemma 2.1; it may be established also by the argument used by Bonsall [3] to prove the Corollary to his Theorem 1. Consequently, if $s_{1}<\xi<s_{2}$ then

$$
\int_{s_{1}}^{s_{2}} y(t) d t \leqq \int_{s_{1}}^{s_{2}} u(t) d t
$$

and the strict inequality holds in (4.7) provided $u(x)$ is strictly sub- $(L)$. From (4.5) it then follows that if $u(x)$ is sub- $(L)$ on $(a, b)$ then

$$
u\left(\xi\left(s_{1}, s_{2}\right)\right) \leqq\left[k\left(s_{1}, s_{2}\right)\right]^{-1} \int_{s_{1}}^{s_{2}} u(t) d t, \quad a<s_{1}<s_{2}<b,
$$

and the strict inequality holds in (4.8) provided $u(x)$ is strictly sub- $(L)$ on $(a, b)$. It is to be commented that an alternate proof of (4.8) for sub- $(L)$ functions $u(x)$ is afforded by the fact that $w_{s_{1} s_{2}}(x) \in \Gamma_{0}^{-}\left(s_{1}, s_{2}\right)$, so that $I\left(w_{s_{1} s_{2}}, u ; s_{1}, s_{2}\right) \geqq 0$ by Theorem 3.1 , and (4.8) results from an integration by parts similar to that used above to establish (4.5).

In order to show that (4.8) actually characterizes sub- $(L)$ functions, 
one needs to prove that if $a<x_{0}<b$ then there exist values $s_{1}, s_{2}$ arbitrarily close to $x_{0}$ and such that $\xi\left(s_{1}, s_{2}\right)=x_{0}$. Now for $z(x ; s)$ the solution of (4.2), and $a<x_{0}<b$, it follows readily that $z\left(x_{0} ; s\right)$ is negative monotone increasing on $a<s<x_{0}$, and negative monotone decreasing on $x_{0}<s<b$. Consequently, if $\delta>0$ is such that

$$
z\left(x_{0} ; x_{0}-\delta\right)>\lim _{s \rightarrow b} z\left(x_{0} ; s\right)
$$

then for $0<h \leqq \delta$ there is a unique value $s_{2}=s_{2}\left(h, x_{0}\right)>x_{0}$ such that $z\left(x_{0} ; s_{2}\right)=z\left(x_{0} ; x_{0}-h\right)$ and $x_{0}=\xi\left(x_{0}-h, s_{2}\left(h, x_{0}\right)\right)$; moreover, $s_{2}\left(h, x_{0}\right) \rightarrow x_{0}$ as $h \rightarrow 0$. If $[c, d]$ is a compact subinterval of $(a, b)$ then clearly the value of $\delta$ satisfying (4.9) may be chosen uniformly for $c \leqq x_{0} \leqq d$.

By indirect argument it will be shown that $u(x)$ is $\operatorname{sub}-(L)$ on $(a, b)$ whenever $u(x)$ is continuous on this interval and the inequality

$$
u(x) \leqq\left[k\left(x-h, s_{2}(h, x)\right)\right]^{-1} \int_{x-h}^{s_{2}(h, x)} u(t) d t, \quad a<x<b,
$$

holds for all $h>0$ such that

$$
z(x ; x-h)>\lim _{s \rightarrow b} z(x ; s) .
$$

Suppose that $u=u_{0}(x)$ is continuous on $(a, b)$ and such that (4.10) holds for all $h>0$ satisfying (4.11), while $u_{0}(x)$ is not sub- $(L)$ on $(a, b)$. Then there exists a subinterval $[c, d]$ such that $y(x)=y\left(x ; c, u_{0}(c) ; d, u_{0}(d)\right)$ satisfies $y(c)=u_{0}(c), y(d)=u_{0}(d), y(x)<u_{0}(x)$ on $(c, d)$. As (4.5) holds for $y(x)$, the function $u(x)=u_{0}(x)-y(x)$ is continuous on $(a, b)$ and satisfies (4.10) with all $h>0$ for which (4.11) holds, while $u(c)=0=u(d), u(x)>0$ on $(c, d)$. If $y_{0}(x)$ is a solution of (2.1) which is positive on $[c, d]$ then the maximum $M$ of $u(x) / y_{0}(x)$ on $[c, d]$ occurs only at interior points of this interval, and hence there exists a point $x_{0} \in(c, d)$ such that $u\left(x_{0}\right) / y_{0}\left(x_{0}\right)=M$, while $u(x) / y_{0}(x) \not \equiv M$ on subintervals $\left[s_{1}, s_{2}\right]$ with $s_{1}<$ $x_{0}<s_{2}$. If $h>0$ is such that $x_{0}-h \geqq c$ and $s_{2}\left(h, x_{0}\right) \leqq d$, then on $\left[x_{0}-h, s_{2}\left(h, x_{0}\right)\right]$ we have $u(x) / y_{0}(x) \leqq M, u(x) / y_{0}(x) \not \equiv M$, and consequently, in view of (4.10),

$$
u\left(x_{0}\right)<\left[k\left(x_{0}-h, s_{2}\left(h, x_{0}\right)\right]^{-1} M \int_{x_{0}-h}^{s_{2}\left(h, x_{0}\right)} y_{0}(t) d t .\right.
$$

As $\xi\left(x_{0}-h, s_{2}\left(h, x_{0}\right)\right)=x_{0}$, however, from (4.6) it follows that the righthand member of (4.12) is equal to $u\left(x_{0}\right)$, and we have the contradiction $u\left(x_{0}\right)<u\left(x_{0}\right)$. Consequently, if $u=u_{0}(x)$ is a continuous function such that (4.10) holds for all $h>0$ satisfying (4.11) then $u_{0}(x)$ is sub- $(L)$ on $(a, b)$. If the strict inequality in (4.10) holds for all $h>0$ satisfying (4.11), then since (4.5) is satisfied by all solutions $y(x)$ of (2.1) it follows that there is no subinterval on which $u(x)$ is a solution of $(2.1)$, so that 
$u(x)$ is strictly sub- $(L)$ on $(a, b)$. We have established, therefore, the following result.

Theorem 4.1. If (2.1) satisfies condition $(I)$ on $(a, b)$, then $a$ necessary and sufficient condition for $u(x)$ to be $\{$ strictly\} sub- $(L)$ on $(a, b)$ is that $u(x)$ be continuous on this interval and the inequality (4.10) holds \{in the strict sense $\}$ for all $h>0$ satisfying (4.11).

It is to be remarked that for the above proof of the sufficient condition in Theorem 4.1 one need not require that (4.10) hold for all $h>0$ satisfying (4.11), but merely that for each $x \in(a, b)$ there is a sequence of such values $h$ approaching zero and for which (4.10) holds.

If $L(y) \equiv y^{\prime \prime}$, then $z(x ; s)=-(x-s)^{2} / 2, \xi\left(s_{1}, s_{2}\right)=\left(s_{1}+s_{2}\right) / 2, s_{2}\left(h, x_{0}\right)=$ $x_{0}+h, k\left(x_{0}-h, x_{0}+h\right)=2 h$, and (4.10) reduces to (4.1).

If $m>0$, and $L(y) \equiv y^{\prime \prime}+m^{2} y$, then $L(y)=0$ satisfies condition (I) on any interval $x_{1}<x<x_{1}+\pi / m$. The corresponding $z(x ; s)$ is equal to $[\cos m(x-s)-1] / m^{2}$ and

$\xi\left(s_{1}, s_{2}\right)=\left(s_{1}+s_{2}\right) / 2, s_{2}\left(h, x_{0}\right)=x_{0}+h, k\left(x_{0}-h, x_{0}+h\right)=(2 / m) \sin m h$,

and $u(x)$ is sub- $(L)$ on a subinterval $\left(a_{1}, b_{1}\right)$ of $x_{1}<x<x_{1}+\pi / m$ if and only if $u(x)$ is continuous and

$$
u(x) \leqq[m /(2 \sin m h)] \int_{x-h}^{x+h} u(t) d t, \quad a_{1}<x<b_{1},
$$

for all $h>0$ such that $a_{1}+h<x<b_{1}-h$.

Correspondingly, if $m>0$ and $L(y) \equiv y^{\prime \prime}-m^{2} y$, then $L(y)=0$ satisfies condition (I) on $(-\infty, \infty)$. The associated $z(x ; s)$ is equal to $[1-\cosh m(x-s)] / m^{2}$ and $\xi\left(s_{1}, s_{2}\right)=\left(s_{1}+s_{2}\right) / 2, \quad s_{2}\left(h, x_{0}\right)=x_{0}+h$, $k\left(x_{0}-h, x_{0}+h\right)=(2 / m)$ sinh $m h$, and $u(x)$ is sub- $(L)$ on an interval $\left(a_{1}, b_{1}\right)$ if and only if $u(x)$ is continuous and

$$
u(x) \leqq[m /(2 \sinh m h)] \int_{x-h}^{x+h} u(t) d t, \quad a_{1}<x<b_{1},
$$

for all $h>0$ satisfying $a_{1}+h<x<b_{1}-h$.

\section{REFERENCES}

1. E. F. Beckenbach, Generalized convex functions, Bull. Amer. Math. Soc., 43 (1937), 363-371.

2. G. A. Bliss, Lectures on the calculus of variations, University of Chicago Press, 1946.

3. F. F. Bonsall, The characierization of generalized convex functions, Quart. J. Math., Oxford Ser. (2), 1 (1950), 100-111.

4. A. B. Carson, An analogue of Green's iteorem for multiple integral problems in the calculus of variaitions, (Dissertation, Chicago, 1941), Contributions to the Calculus of Variations, Chicago (1938-1941), 453-489.

5. J. W. Green, Support, convergence, and differenliability properties of generalizcd 
convex functions, Proc. Amer. Math. Soc., 4 (1953), 391-396.

6. J. H. Levin, Minima of double iniegrals with respect to unilateral variaitions and applications to sub-harmonic funciions, (Dissertation, Chicago, 1940), Contributions to the Calculus of Variations, Chicago (1938-1941), 357-411.

7. M. Morse, The calculus of variations in the large, Amer. Math. Coll. Publ. XVIII, (1934).

8. M. M. Peixoto, Generalized convex funciions and second order differential inequalities, Bull. Amer. Math. Soc., 55 (1949), 563-572.

UNIVER ITY OF CALIForNia, LOS ANGELES AND

NORTHWESTERN UNIVERSITY 



\section{PACIFIC JOURNAL OF MATHEMATICS}

\section{EDITORS}

\section{David Gilbarg}

Stanford University

Stanford, California

\section{R. A. Beaumont}

University of Washington

Seattle 5 , Washington

\author{
A. L. Whiteman
}

University of Southern California Los Angeles 7, California

L. J. Paige

University of California

Los Angeles 24, California

\author{
E. F. BECKENBACH \\ C. E. BURGESS \\ E. HEWITT \\ A. HORN
}

\author{
V. GANAPATHY IYER \\ R. D. JAMES \\ M. S. KNEBELMAN \\ L. NACHBIN
}

ASSOCIATE EDITORS
I. NIVEN

T. G. OSTROM

H. L. ROYDEN

M. M. SCHIFFER
E. G. STRAUS

G. SZEKERES

F. WOLF

K. YOSIDA

\section{SUPPORTING INSTITUTIONS}

\author{
UNIVERSITY OF BRITISH COLUMBIA \\ CALIFORNIA INSTITUTE OF TECHNOLOGY \\ UNIVERSITY OF CALIFORNIA \\ MONTANA STATE UNIVERSITY \\ UNIVERSITY OF NEVADA \\ OREGON STATE COLLEGE \\ UNIVERSITY OF OREGON \\ OSAKA UNIVERSITY \\ UNIVERSITY OF SOUTHERN CALIFORNIA
}

\author{
STANFORD UNIVERSITY \\ UNIVERSITY OF TOKYO \\ UNIVERSITY OF UTAH \\ WASHINGTON STATE COLLEGE \\ UNIVERSITY OF WASHINGTON \\ * * * \\ AMERICAN MATHEMATICAL SOCIETY \\ CALIFORNIA RESEARCH CORPORATION \\ HUGHES AIRCRAFT COMPANY \\ SPACE TECHNOLOGY LABORATORIES
}

Mathematical papers intended for publication in the Pacific Journal of Mathematics should be typewritten (double spaced), and the author should keep a complete copy. Manuscripts may be sent to any one of the four editors. All other communications to the editors should be addressed to the managing editor, L. J. Paige at the University of California, Los Angeles 24, California.

50 reprints per author of each article are furnished free of charge; additional copies may be obtained at cost in multiples of 50 .

The Pacific Journal of Mathematics is published quarterly, in March, June, September, and December. The price per volume (4 numbers) is $\$ 12.00$; single issues, $\$ 3.50$. Back numbers are available. Special price to individual faculty members of supporting institutions and to individual members of the American Mathematical Society: $\$ 4.00$ per volume; single issues, $\$ 1.25$.

Subscriptions, orders for back numbers, and changes of address should be sent to Pacific Journal of Mathematics, 2120 Oxford Street, Berkeley 4, California.

Printed at Kokusai Bunken Insatsusha (International Academic Printing Co., Ltd.), No. 6, 2-chome, Fujimi-cho, Chiyoda-ku, Tokyo, Japan.

PUBLISHED BY PACIFIC JOURNAL OF MATHEMATICS, A NON-PROFIT CORPORATION

The Supporting Institutions listed above contribute to the cost of publication of this Journal, but they are not owners or publishers and have no responsibility for its content or policies. 


\section{Pacific Journal of Mathematics}

\section{Vol. 9, No. $2 \quad$ June, 1959}

Lee William Anderson, On the breadth and co-dimension of a topological lattice

Frank W. Anderson and Robert L. Blair, Characterizations of certain lattices

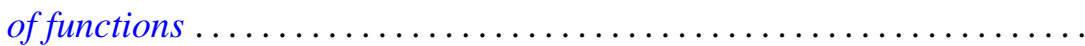

Donald Charles Benson, Extensions of a theorem of Loewner on integral

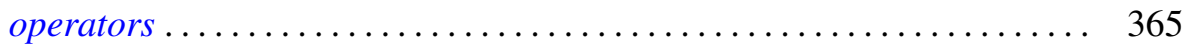

Errett Albert Bishop, A duality theorem for an arbitrary operator ........ 379

Robert McCallum Blumenthal and Ronald Kay Getoor, The asymptotic distribution of the eigenvalues for a class of Markov operators ........

Delmar L. Boyer and Elbert A. Walker, Almost locally pure Abelian

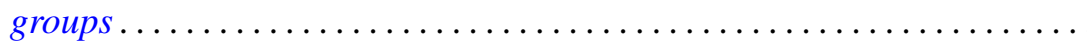

Paul Civin and Bertram Yood, Involutions on Banach algebras ........... Lincoln Kearney Durst, Exceptional real Lehmer sequences .... 415

Eldon Dyer and Allen Lowell Shields, Connectivity of topological

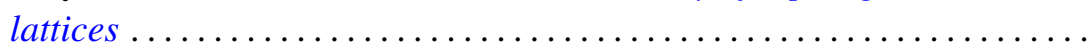

Ronald Kay Getoor, Markov operators and their associated

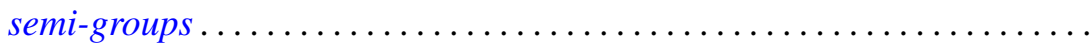

Bernard Greenspan, A bound for the orders of the components of a system of algebraic difference equations

Branko Grünbaum, On some covering and intersection properties in

Minkowski spaces ............................

Bruno Harris, Derivations of Jordan algebras ..............

Henry Berge Helson, Conjugate series in several variables.

Isidore Isaac Hirschman, Jr., A maximal problem in harmonic analysis.

II .

Alfred Horn and Robert Steinberg, Eigenvalues of the unitary part of a matrix

Edith Hirsch Luchins, On strictly semi-simple Banach algebras ...

William D. Munro, Some iterative methods for determining zeros of

functions of a complex variable...

John Rainwater, Spaces whose finest uniformity is metric .

William T. Reid, Variational aspects of generalized convex functions ....

A. Sade, Isomorphisme d'hypergroupoï des isotopes ...... . .

Isadore Manual Singer, The geometric interpretation of a special

connection . . .

Charles Andrew Swanson, Asymptotic perturbation series for characteristic

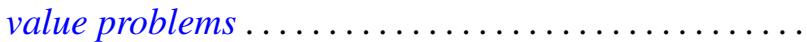

